\title{
Comparison of the Efficacy and Safety of Insulin Detemir Administered Once Daily According to Two Titration Algorithms (3-0-3 and 2-4-6-8) in Patients with Type 2 Diabetes Mellitus
}

Hea Min $\mathrm{Yu}^{1}$, Kang Seo Park ${ }^{1}$, Jun Hwa Hong ${ }^{1}$, Keun Yong Park ${ }^{2}$, Jong Min Lee ${ }^{3}$, Bon Jeong Ku ${ }^{4}$, Yeo Joo Kim Tae Kun $\mathrm{Oh}^{6}$

${ }^{1}$ Division of Endocrinology and Metabolism, Department of Internal Medicine, Daejeon Eulji Medical Center, Eulji University School of Medicine; ${ }^{2}$ Department of Endocrinology and Metabolism, Konyang University Hospital, Konyang University College of Medicine; ${ }^{3}$ Department of Endocrinology and Metabolism, Daejeon St. Mary's Hospital, College of Medicine, The Catholic University of Korea; ${ }^{4}$ Department of Endocrinology and Metabolism, Chungnam National University Hospital, Chungnam National University College of Medicine, Daejeon; ${ }^{5}$ Department of Endocrinology and Metabolism, Soonchunhyang University Cheonan Hospital, Soonchunhyang University College of Medicine, Cheonan; ${ }^{6}$ Department of Endocrinology and Metabolism, Chungbuk National University Hospital, Chungbuk National University College of Medicine, Cheongju, Korea

Background: This study was conducted to compare glycaemic control with insulin detemir administered according to two titration algorithms (3-0-3 and 2-4-6-8) after 20 weeks of treatment in subjects with type 2 diabetes mellitus inadequately controlled on metformin. Methods: This was a 20-week, randomised, multicentre, open-labelled, treat-to-target trial. Forty-six patients were randomised in a 1:1 manner to either the 3-0-3 (G3, n=23) or 2-4-6-8 (G2, n=23) algorithm. The primary endpoint was change of haemoglobin A1c (HbA1c), and the secondary safety endpoint included hypoglycaemic events.

Results: After 20 weeks, HbA1c decreased similarly in the G3 and G2 groups, with a mean change of $-0.9 \%$ from baseline. The mean change in fasting plasma glucose was numerically similar in both groups. The hypoglycaemia event rate per 100-patient-years of exposure $(r)$ in the $\mathrm{G} 2$ group $(r=1,427)$ was higher than that in the G3 group $(r=807)$.

Conclusion: Both treatment groups had numerically similar HbA1c reductions. A trend towards fewer hypoglycaemia episodes after dose stabilisation was seen with the simpler G3. Clinically, this may be an important observation, as a simpler titration algorithm may support self-management and maintenance of insulin therapy.

Keywords: Hyperglycemia; Insulin; Algorithms; Diabetes mellitus, type 2

\section{INTRODUCTION}

Type 2 diabetes mellitus (T2DM) has become a global health problem. Nearly 415 million people worldwide are estimated to be affected by T2DM as of 2015, a number that is projected to increase to 642 million by 2040 [1]. T2DM is associated with
Received: 22 October 2019, Revised: 2 January 2020, Accepted: 13 January 2020 Corresponding author: Kang Seo Park

Division of Endocrinology and Metabolism, Department of Internal Medicine, Daejeon Eulji Medical Center, Eulji University School of Medicine, 95 Dunsanseo-ro, Seo-gu, Daejeon 35233, Korea

Tel: +82-42-611-3000, Fax: +82-42-259-1162, E-mail: pkkss@eulji.ac.kr
Copyright $\odot 2020$ Korean Endocrine Society

This is an Open Access article distributed under the terms of the Creative Commons Attribution Non-Commercial License (https://creativecommons.org/ licenses/by-nc/4.0/) which permits unrestricted non-commercial use, distribution, and reproduction in any medium, provided the original work is properly cited. 
the progressive deterioration of $\beta$-cell mass and function with consequent depletion of insulin secretion and failure to maintain target haemoglobin A1c (HbA1c) levels, leading to various complications.

The addition of long-acting basal insulin analogues to existing oral antidiabetic drugs (OADs) has been shown to achieve clinically beneficial improvements in HbA1c levels [1-4].

Insulin detemir (Levemir, Novo Nordisk, Bagsvaerd, Denmark) is a long-acting soluble insulin analogue that was developed to enable subjects with diabetes mellitus to maintain more stable glycaemic levels with relatively low day-to-day variability [5]. An international observational study (Study of Once Daily Levemir) supported the use of insulin detemir as a basal insulin analogue when initiating insulin therapy in patients with T2DM [6]. Furthermore, a once daily regimen of insulin detemir was shown to be as effective as a twice-daily regimen [6-8].

Glycaemic control remains suboptimal in many diabetic populations. Inappropriate titration is one of the major reasons for suboptimal insulin doses, resulting in failure to achieve target glycaemic levels $[9,10]$. Self-titration regimens could facilitate patient empowerment, allowing patients to be involved in the treatment plan and thereby helping them achieve target glycaemic levels. Patient-directed titration of insulin has demonstrated HbA1c decreases of approximately $2.5 \%$ [11]. Two titration algorithms of insulin detemir are recommended for adults with T2DM. The 2-4-6-8 titration algorithm was approved by the European Medicines Agency (EMA) in 2007 and by the Korean Ministry of Food and Drug Safety (MFDS) in 2011. The 3-0-3 titration algorithm is simpler, with efficacy and safety profiles similar to those of the 2-4-6-8 algorithm [11]. It was approved for adult T2DM by the EMA in 2014. Early large cohort studies with insulin detemir reported that simple, yet effective 3-0-3 titration algorithm empowered patients to be active participants in their treatment plan, leading to greater glycaemic control $[5,11]$. The 3-0-3 algorithm may also be more appropriate for patients with a higher body mass index (BMI), who generally require a higher dose of insulin to achieve optimum glycaemic control. However, studies of real-world experiences of using the 3-0-3 algorithm of insulin detemir have been particularly lacking in Asian patients with T2DM inadequately controlled with OADs.

The present trial, for the first time, compared the efficacy and safety of two titration algorithms of insulin detemir (2-4-6-8 and 3-0-3) administered once daily in adult patients with T2DM inadequately controlled by metformin, with or without other OADs, in Korea.

\section{METHODS}

\section{Ethical considerations}

This protocol was approved by the Institutional Review Boards of Eulji University School of Medicine (IRB No. 2013-04-009), Konyang University Hospital, Daejeon St. Mary’s Hospital, College of Medicine, The Catholic University of Korea, Chungnam National University Hospital, Soonchunhyang University Cheonan Hospital, and Chungbuk National University Hospital. The study was conducted in accordance with the Declaration of Helsinki and guidelines on good clinical practice. All participants provided written informed consent (ClinicalTrials.gov Identifier: NCT01868542).

\section{Study participants and study design}

A 20-week, randomised (1:1), multicentre, open-labelled, parallel-group, treat-to-target trial was conducted to compare the efficacy and safety of two titration algorithms (3-0-3 and 2-4-6-8) of insulin detemir (Levemir) administered once daily in subjects with T2DM inadequately controlled by metformin treatment in Korea. The randomisation was carried out in a 1:1 manner using an electronic data capture application.

Study participants consisted of insulin-naive men and women with T2DM. The inclusion criteria were as follows: age $\geq 18$ years, diagnosed with T2DM at least 3 months prior to visit 1, treatment with at least $1,000 \mathrm{mg}$ of metformin per day with or without other OADs at a stable dose (at either the maximum tolerated dose or at least half of the maximum recommended dose according to the package insert) for at least 3 months prior to visit $1, \mathrm{HbA} 1 \mathrm{c} \geq 7.5 \%$ by a central laboratory analysis, and BMI $\leq 35.0 \mathrm{~kg} / \mathrm{m}^{2}$. Patients with the following conditions were excluded: female patients of child-bearing potential who were pregnant or breastfeeding, intended to become pregnant, or were not using adequate contraceptive methods; the receipt of any investigational product within 4 weeks prior to visit 1 , any contraindication to insulin detemir according to the domestic labelling; the anticipated change of dose of any systemic treatment with products that in the investigator's opinion could interfere with glucose metabolism (such as systemic corticosteroids, $\beta$-blockers, or monoamine oxidase inhibitors); clinically significant diseases that, in the investigator's opinion, could potentially confound the results of the trial or pose additional risk in the administration of the trial product; or any other condition that the investigator determined would interfere with trial participation or evaluation of the results.

At visit 1, demographic information and a clinical history 


\begin{tabular}{|lcc|}
\hline $\begin{array}{l}\text { Table 1. Insulin Detemir Titration Algorithms } \\
\text { Algorithm for dose } \\
\text { adjustment }\end{array}$ & $\begin{array}{c}\text { The lowest of } \\
\text { S pre-breakfast } \\
\text { SMPG values, } \mathrm{mmol} / \mathrm{L}\end{array}$ & $\begin{array}{c}\text { Insulin detemir } \\
\text { dose adjustment }\end{array}$ \\
\hline 3-0-3 algorithm [11] & $>6.1(>110 \mathrm{mg} / \mathrm{dL})$ & $+3 \mathrm{U}$ \\
$4.4-6.1(80-110 \mathrm{mg} / \mathrm{dL})$ & No adjustment \\
& $<4.4(<80 \mathrm{mg} / \mathrm{dL})$ & $-3 \mathrm{U}$ \\
& $>10.0(180 \mathrm{mg} / \mathrm{dL})$ & $+8 \mathrm{U}$ \\
$9.1-10.0(163-180 \mathrm{mg} / \mathrm{dL})$ & $+6 \mathrm{U}$ \\
$8.1-9.0(145-162 \mathrm{mg} / \mathrm{dL})$ & $+4 \mathrm{U}$ \\
$7.1-8.0(127-144 \mathrm{mg} / \mathrm{dL})$ & $+2 \mathrm{U}$ \\
$6.1-7.0(109-126 \mathrm{mg} / \mathrm{dL})$ & $+2 \mathrm{U}$ \\
& $4.1-6.0(73-108 \mathrm{mg} / \mathrm{dL})$ & No adjustment \\
& $3.1-4.0(56-72 \mathrm{mg} / \mathrm{dL})$ & $-2 \mathrm{U}$ \\
& $<3.1(<56 \mathrm{mg} / \mathrm{dL})$ & $-4 \mathrm{U}$ \\
\hline
\end{tabular}

were obtained, a physical examination was performed, and eligibility was evaluated. At the randomisation visit (visit 2), which took place a maximum of 2 weeks after the screening visit, patients were allocated to one of the two titration groups. The randomisation visit was followed by a titration period of 20 weeks. Insulin doses were adjusted depending on the lowest of 3 pre-breakfast self-monitored plasma glucose (SMPG) readings. The treatment algorithms are outlined in Table 1.

\section{Efficacy parameters}

The primary endpoint was the change of $\mathrm{HbAlc}(\%)$ from baseline after 20 weeks of treatment, which was summarized descriptively according to the titration algorithm. Secondary endpoints included the change in $\mathrm{HbAlc}$ at 12 weeks of treatment after visit 2 , change in fasting plasma glucose (FPG) from baseline to 12 and 20 weeks of treatment, and glycaemic control measured by 7-point SMPG profiles at 20 weeks of treatment (before and 90 minutes after breakfast, lunch, and dinner, and at bedtime).

\section{Safety parameters}

The safety parameters included hypoglycaemic episodes, the average insulin dose during the trial for all subjects, change in body weight at 20 weeks of treatment from visit 2 (week 0 ), and adverse events (AEs) and other safety findings after 20 weeks of treatment. Vital signs and standard lab parameters (haematol- ogy, biochemistry, urinalysis, and lipids) were assessed. Hypoglycaemic episodes were classified according to the American Diabetes Association (ADA) definition (severe, documented symptomatic, asymptomatic, probable symptomatic, and relative). Nocturnal hypoglycaemic episodes were determined by the ADA definition. AEs were coded using version 17 of the Medical Dictionary for Regulatory Activities.

\section{Statistical methods}

This was planned as a non-inferiority trial with a non-inferiority margin of $0.4 \%$ as per the Food and Drug Administration (FDA). The planned total number of randomised subjects was to be at least 236, but the trial was terminated with only 46 randomised subjects due to a low recruitment rate. A post hoc decision was taken not to conduct any planned statistical analyses. Therefore, descriptive statistics of each endpoint are presented in the Results section. The pull analysis set, per-protocol analysis set, and safety analysis set were defined in accordance with the International Conference on Harmonization (ICH) E9 guidance. Furthermore, a subgroup analysis of the safety population was conducted. The date of visit 14 was used as the cut-off date that differentiated the period of baseline to 12 weeks from the period of 12 weeks to the end of treatment (EOT). The McNemar exact test was used to test the significance of changes in the occurrence of hypoglycaemic episodes and nocturnal hypoglycaemic episodes from baseline to 12 weeks versus from 12 weeks to EOT within each treatment group. The chi-square test was used to test the significance of differences in the rates of hypoglycaemic episodes and nocturnal hypoglycaemic episodes between treatment groups for each specific period of time.

\section{RESULTS}

\section{Subject disposition}

A total of 58 subjects were screened, and 12 subjects were excluded on the basis of the screening. A total of 46 subjects were randomised, and 44 subjects completed the trial.

\section{Baseline characteristics}

In general, the demographics and baseline characteristics were similar between the two groups, with only marginal differences. The study population consisted of Asian men and women with T2DM (nine male patients [39.1\%] in the 3-0-3 algorithm group and 12 male patients [52.2\%] in the 2-4-6-8 algorithm group), and had a mean age of 56.1 years (ranging, 36 to 75), a mean height of $1.62 \mathrm{~m}$ (range, 1.46 to 1.85 ), a mean body weight of 


\begin{tabular}{|c|c|c|c|}
\hline Characteristic & $\begin{array}{c}3-0-3 \\
\text { algorithm } \\
(n=23)\end{array}$ & $\begin{array}{c}2-4-6-8 \\
\text { algorithm } \\
(n=21)\end{array}$ & Total \\
\hline Age (mean), yr & $54.4 \pm 12.0$ & $57.7 \pm 6.3$ & $56.1 \pm 9.7$ \\
\hline Height, m & $1.59 \pm 0.10$ & $1.65 \pm 0.08$ & $1.62 \pm 0.09$ \\
\hline Body weight, $\mathrm{kg}$ & $64.5 \pm 15.1$ & $67.4 \pm 11.3$ & $65.6 \pm 13.2$ \\
\hline BMI, $\mathrm{kg} / \mathrm{m}^{2}$ & $25.2 \pm 4.0$ & $24.7 \pm 3.1$ & $24.8 \pm 3.5$ \\
\hline Duration of diabetes, yr & $12.5 \pm 7.3$ & $15.9 \pm 52.6$ & $13.3 \pm 6.5$ \\
\hline HbA1c, $\%$ & $10.1 \pm 1.6$ & $9.6 \pm 0.9$ & $9.7 \pm 1.3$ \\
\hline \multicolumn{4}{|l|}{ All medications } \\
\hline Metformin $+\mathrm{SU}$ & 0 & $3(13.0)$ & $3(6.5)$ \\
\hline Metformin+SU+DPP-4i & $22(95.7)$ & $17(73.9)$ & $39(84.8)$ \\
\hline $\begin{array}{l}\text { Metformin+SU+DPP- } \\
\quad 4 \mathrm{i}+\alpha-\mathrm{GI}\end{array}$ & $1(4.3)$ & $1(4.3)$ & $2(4.3)$ \\
\hline Metformin $+\mathrm{SU}+\alpha-\mathrm{GI}$ & 0 & $1(4.3)$ & $1(2.2)$ \\
\hline $\begin{array}{l}\text { Metformin }+\mathrm{SU}+\alpha- \\
\text { GI+TZD }\end{array}$ & 0 & $1(4.3)$ & $1(2.2)$ \\
\hline
\end{tabular}

Values are expressed as mean \pm standard deviation or number (\%). BMI, body mass index; HbA1c, haemoglobin A1c; SU, sulphonylurea; DPP-4i, dipeptidyl peptidase 4 inhibitor; $\alpha$-GI, $\alpha$-glucosidase-inhibitor; TZD, thiazolidinedione.

$65.6 \mathrm{~kg}$ (range, 43.0 to 103.5 ), a mean BMI of $24.8 \mathrm{~kg} / \mathrm{m}^{2}$ (range, 18.3 to 32.8 ), a mean duration of diabetes of 13.3 years (range, 1.3 to 31.6 ), and a mean $\mathrm{HbA} 1 \mathrm{c}$ of $9.7 \%$ (range, $7.3 \%$ to $14.8 \%$ ) (Table 2).

\section{Change in $\mathrm{HbA1c}(\%)$ from baseline to 20 weeks of treatment}

Mean HbAlc levels decreased during the trial, and the most prominent reduction was observed during the first 12 weeks of the trial. The mean change in HbA1c from baseline to week 12 was $-0.8 \%$ in the $3-0-3$ algorithm group and $-0.9 \%$ in the $2-4-$ 6-8 algorithm group. The mean change in HbA1c from baseline to week 20 was identical $(-0.9 \%)$ in both treatment algorithm groups (Fig. 1).

\section{Change in FPG from baseline to 12 and 20 weeks of treatment}

A steady decrease in the FPG was observed in the 2-4-6-8 algorithm group, whereas the FPG decreased for the first 12 weeks and then increased by the end of week 20 in the 3-0-3 algorithm group. The mean change in FPG was greater in the 2-4-6-8 algorithm group ( $-74.3 \mathrm{mg} / \mathrm{dL})$ than in the 3-0-3 algorithm group $(-44.6 \mathrm{mg} / \mathrm{dL})$ after 12 weeks of treatment.

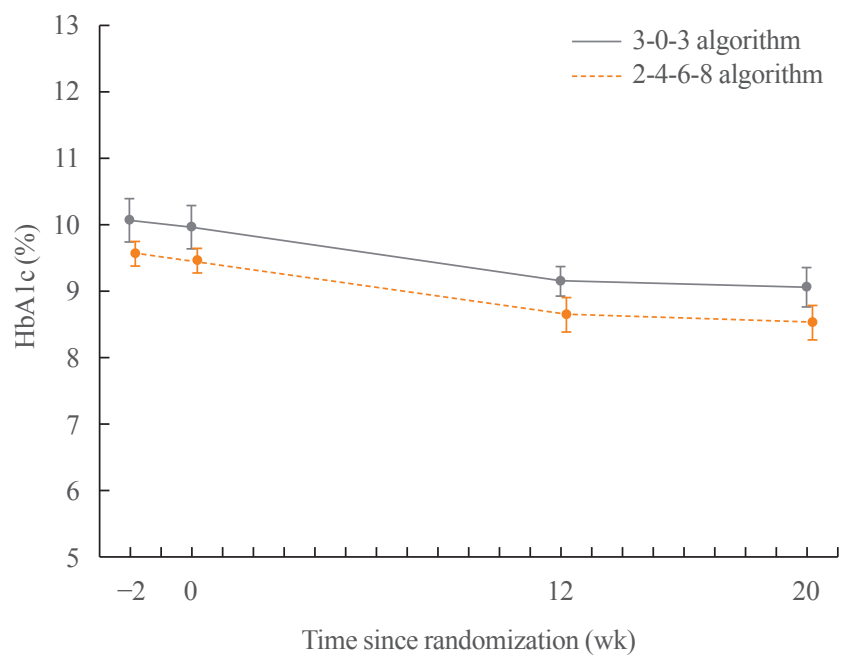

Fig. 1. Mean haemoglobin A1c (HbA1c) reduction by week during the trial. Changes in $\mathrm{HbA} 1 \mathrm{c}$ from baseline to 20 weeks of treatment: HbA1c decreased similarly in the 3-0-3 algorithm group $(-0.9 \%$ from $10.0 \%$ at baseline) and in the 2-4-6-8 algorithm group (-0.9\% from $9.5 \%$ at baseline) after 20 weeks of treatment.

The mean change in FPG was similar for both treatment algorithms after 20 weeks of treatment: $-60.4 \mathrm{mg} / \mathrm{dL}$ in the $3-0-3$ algorithm group and $-56.4 \mathrm{mg} / \mathrm{dL}$ in the $2-4-6-8$ algorithm group (Fig. 2).

\section{Seven-point SMPG profiles (before and 90 minutes after breakfast, lunch and dinner, and at bedtime) at 20 weeks of treatment}

The 7-point SMPG profiles showed slight improvements after 20 weeks of treatment in both treatment algorithm groups. The decrease in the mean 7-point SMPG was numerically greater in the 2-4-6-8 algorithm group (68.4 $\mathrm{mg} / \mathrm{dL}$ ) than in the 3-0-3 algorithm group $(59.9 \mathrm{mg} / \mathrm{dL})$ after 20 weeks of treatment. The prandial increment for all meals was observed to increase more in the 3-0-3 algorithm group than in the 2-4-6-8 algorithm group.

\section{Safety results}

The hypoglycaemia event rate per 100-patient-years of exposure $(r)$ in the 2-4-6-8 algorithm was higher than that in the 3-03 algorithm group. No severe hypoglycaemic episodes were reported in either group. A further sub-analysis evaluating overall hypoglycaemic episodes from 0 to 12 weeks and from 12 weeks to EOT revealed significantly fewer episodes in the 3-0-3 algorithm group ( 9 vs. $16, P<0.05$ ) from 12 weeks to EOT, with no differences in the occurrence of nocturnal hypoglycaemia (Table 
3). The average insulin dose was observed to increase slightly more in the 3-0-3 algorithm group (Table 4), and patient's mean body weight increased more in the 3-0-3 group $(2.1 \mathrm{~kg})$ than in the 2-4-6-8 algorithm group $(0.8 \mathrm{~kg})$ after 20 weeks of treatment. There were no clinically relevant differences between the treatment algorithm groups in reported AEs. A similar percentage of subjects reported AEs in the 3-0-3 (52.2\%) and 2-4-6-8 $(56.5 \%)$ algorithm groups. The most frequently reported AEs in both groups were nasopharyngitis, upper respiratory tract infection, headache, and rash. All AEs were either mild or moderate

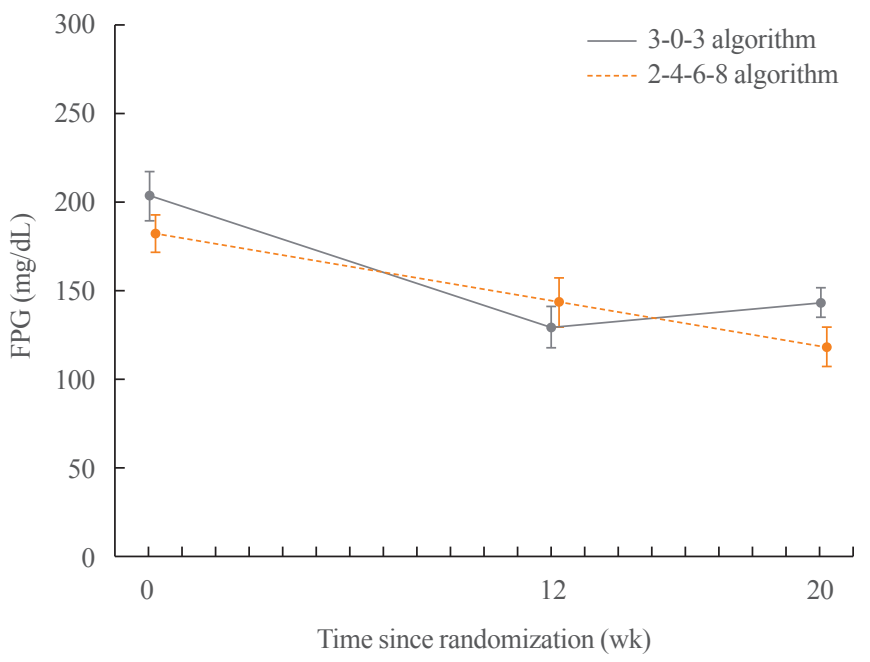

Fig. 2. Mean fasting plasma glucose (FPG) values by week during the trial. FPG was observed to decrease more in the 2-4-6-8 algorithm group $(-74.3 \mathrm{mg} / \mathrm{dL})$ than in the 3-0-3 algorithm group ( -44.6 $\mathrm{mg} / \mathrm{dL}$ ) after 12 weeks of treatment. FPG decreased similarly in the 3-0-3 algorithm group ( $-60.4 \mathrm{mg} / \mathrm{dL}$ from $204.1 \mathrm{mg} / \mathrm{dL}$ at baseline) and in the 2-4-6-8 algorithm group $(-56.4 \mathrm{mg} / \mathrm{dL}$ from $182.9 \mathrm{mg} / \mathrm{dL}$ at baseline) after 20 weeks of treatment. in severity. No deaths were reported in this trial.

\section{DISCUSSION}

This is the first randomised trial to compare the efficacy and safety of two titration algorithms (3-0-3 and 2-4-6-8 algorithms) of insulin detemir (Levemir) in Korean subjects with T2DM inadequately controlled by metformin. Insulin detemir is a longacting basal insulin analogue that has been approved by the EMA, FDA, the Therapeutic Goods Administration, Health Canada, and most other authorities for the treatment of diabetes mellitus in combination with OADs and as part of basal-bolus insulin regimens. Due to its lower within-subject variability profile and the more predictable glycaemic response that it provides, insulin detemir enables subjects with diabetes mellitus to

\begin{tabular}{|c|c|c|c|}
\hline & $\begin{array}{c}3-0-3 \\
\text { algorithm } \\
(n=23)\end{array}$ & $\begin{array}{c}2-4-6-8 \\
\text { algorithm } \\
(n=23)\end{array}$ & $P$ value \\
\hline \multicolumn{4}{|c|}{ Baseline to EOT } \\
\hline Episode & $15(65.22)$ & $21(91.30)$ & 0.0320 \\
\hline \multicolumn{4}{|c|}{ Baseline to 12 weeks } \\
\hline Episode & $13(56.52)$ & $18(78.26)$ & 0.1158 \\
\hline \multicolumn{4}{|c|}{12 weeks to EOT } \\
\hline Episode & $9(39.13)$ & $16(69.57)$ & 0.0383 \\
\hline
\end{tabular}

Table 4. Changes in the Last Dose of Insulin Taken Prior to Titration

\begin{tabular}{|c|c|c|c|c|c|c|c|}
\hline \multirow{3}{*}{ Outcomes } & \multirow{3}{*}{ Treatment group } & \multirow{3}{*}{ No. } & \multicolumn{5}{|c|}{ Change from baseline to EOT } \\
\hline & & & \multirow{2}{*}{ Mean \pm SD } & \multirow{2}{*}{$\mathrm{LS}$ mean $\pm \mathrm{SE}$} & \multicolumn{3}{|c|}{ Difference mean } \\
\hline & & & & & $\mathrm{LS}$ mean $\pm \mathrm{SE}$ & $95 \% \mathrm{CI}$ & $P$ value \\
\hline \multirow[t]{2}{*}{ Insulin dose, unit } & $3-0-3$ & 23 & $26.61 \pm 28.87$ & $25.40 \pm 5.26$ & & & \\
\hline & $2-4-6-8$ & 21 & $19.24 \pm 20.10$ & $20.68 \pm 5.47$ & $-4.72 \pm 7.73$ & -20.34 to 10.91 & 0.5452 \\
\hline \multirow[t]{2}{*}{ Insulin dose, units $/ \mathrm{kg}$} & $3-0-3$ & 23 & $0.35 \pm 0.38$ & $0.33 \pm 0.07$ & & & \\
\hline & $2-4-6-8$ & 21 & $0.27 \pm 0.29$ & $0.29 \pm 0.08$ & $-0.04 \pm 0.11$ & -0.25 to 0.18 & 0.7285 \\
\hline \multirow[t]{2}{*}{ Insulin dose, units $/ \mathrm{m}^{2}$} & $3-0-3$ & 23 & $14.40 \pm 15.62$ & $13.64 \pm 2.93$ & & & \\
\hline & $2-4-6-8$ & 21 & $10.73 \pm 11.54$ & $11.56 \pm 3.04$ & $-2.08 \pm 4.30$ & -10.78 to 6.61 & 0.6309 \\
\hline
\end{tabular}

The average insulin dose was observed to increase slightly more in the 3-0-3 algorithm group than in the 2-4-6-8 algorithm group. The models included treatment group and sex as fixed effects and age as a covariate.

EOT, end of treatment; SD, standard deviation; LS, least squares; SE, standard error; CI, confidence interval. 
achieve target glycaemic levels without increasing the risk of hypoglycaemia [11]. The results of the Treat to target with once daily Insulin Therapy: Reduce A1C by Titrating Effectively (TITRATE) study showed that a simple, patient-directed titration algorithm empowered patients to adjust their basal insulin dose and thereby achieve measurable improvements in their glycaemic profile [11]. The 2-4-6-8 titration algorithm of insulin detemir was approved by the EMA in 2007 and later by the Korean MFDS. A simpler and similarly effective 3-0-3 algorithm was subsequently approved by the EMA for use in in adults with T2DM. Nonetheless, there is a lack of clinical evidence from real-world settings regarding the effectiveness of the 3-0-3 algorithm of insulin detemir in patients with T2DM. The participants enrolled in the present trial were Korean subjects with T2DM inadequately controlled by metformin with or without other OADs. HbA1c is routinely used to measure glycaemic control when monitoring and guiding therapy. More importantly, HbA1c values predict the risk of microvascular complications, and lowering $\mathrm{HbA} 1 \mathrm{c}$ levels significantly reduces the rate of progression of microvascular complications [12,13]. In this study, both the treatment groups had numerically similar HbA1c reductions after 20 weeks of treatment. Furthermore, during the first 12 weeks in the 3-0-3 algorithm group, a rapid reduction in FPG was observed, without increased hypoglycaemia episodes. Patients with T2DM often delay insulin therapy due to fears of injections and hypoglycaemia. A trend towards fewer hypoglycaemia episodes post-dose stabilisation was seen with the simpler 3-0-3 algorithm. Clinically, this may be important, as a simpler titration algorithm may support self-management and maintenance of insulin therapy with similar glycaemic control and fewer hypoglycaemia episodes. The current study does, however, have limitations. The investigators decided to stop the trial due to a low recruitment rate despite various efforts to recruit subjects. Due to the limited number of patients, no definitive conclusions can be drawn.

In conclusion, in the present trial from Korea, it was observed that the simple 3-0-3 titration algorithm of once daily insulin detemir in patients with T2DM inadequately controlled on metformin treatment with or without other OADs yielded similar results to the 2-4-6-8 titration algorithm for efficacy in terms of the reduction in $\mathrm{HbA} 1 \mathrm{c}$ levels, with fewer hypoglycaemic episodes.

\section{CONFLICTS OF INTEREST}

This study was undertaken as an investigator-initiated research protocol and was funded by Novo Nordisk.

\section{AUTHOR CONTRIBUTIONS}

Conception or design: K.S.P., K.Y.P., J.M.L., B.J.K., Y.J.K., T.K.O. Acquisition, analysis, or interpretation of data: K.S.P., K.Y.P., J.M.L., B.J.K., Y.J.K., T.K.O. Drafting the work or revising: H.M.Y., K.S.P., J.H.H. Final approval of the manuscript: H.M.Y., K.S.P.

\section{ORCID}

Hea Min Yu https://orcid.org/0000-0001-9731-955X

Kang Seo Park https://orcid.org/0000-0002-7964-804X

\section{REFERENCES}

1. Home P, Naggar NE, Khamseh M, Gonzalez-Galvez G, Shen C, Chakkarwar P, et al. An observational non-interventional study of people with diabetes beginning or changed to insulin analogue therapy in non-Western countries: the A1chieve study. Diabetes Res Clin Pract 2011;94:352-63.

2. Riddle MC, Rosenstock J, Gerich J; Insulin Glargine 4002 Study Investigators. The treat-to-target trial: randomized addition of glargine or human NPH insulin to oral therapy of type 2 diabetic patients. Diabetes Care 2003;26:3080-6.

3. Philis-Tsimikas A, Charpentier G, Clauson P, Ravn GM, Roberts VL, Thorsteinsson B. Comparison of once-daily insulin detemir with NPH insulin added to a regimen of oral antidiabetic drugs in poorly controlled type 2 diabetes. Clin Ther 2006;28:1569-81.

4. Jabbour S. Primary care physicians and insulin initiation: multiple barriers, lack of knowledge or both? Int J Clin Pract 2008;62:845-7.

5. Fajardo Montanana C, Hernandez Herrero C, Rivas Fernandez M. Less weight gain and hypoglycaemia with once-daily insulin detemir than NPH insulin in intensification of insulin therapy in overweight type 2 diabetes patients: the PREDICTIVE BMI clinical trial. Diabet Med 2008;25:91623.

6. Caputo S, Andersen H, Kaiser M, Karnieli E, Meneghini LF, Svendsen AL, et al. Effect of baseline glycosylated hemoglobin A1c on glycemic control and diabetes management following initiation of once-daily insulin detemir in real-life clinical practice. Endocr Pract 2013;19:462-70.

7. Hollander P, Raslova K, Skjoth TV, Rastam J, Liutkus JF. Efficacy and safety of insulin detemir once daily in combination with sitagliptin and metformin: the TRANSITION 
randomized controlled trial. Diabetes Obes Metab 2011;13: 268-75.

8. Le Floch JP, Levy M, Mosnier-Pudar H, Nobels F, Laroche $\mathrm{S}$, Gonbert S, et al. Comparison of once- versus twice-daily administration of insulin detemir, used with mealtime insulin aspart, in basal-bolus therapy for type 1 diabetes: assessment of detemir administration in a progressive treat-to-target trial (ADAPT). Diabetes Care 2009;32:32-7.

9. Davies M, Storms F, Shutler S, Bianchi-Biscay M, Gomis R; ATLANTUS Study Group. Improvement of glycemic control in subjects with poorly controlled type 2 diabetes: comparison of two treatment algorithms using insulin glargine. Diabetes Care 2005;28:1282-8.

10. Holman RR, Paul SK, Bethel MA, Matthews DR, Neil HA. 10-Year follow-up of intensive glucose control in type 2 diabetes. N Engl J Med 2008;359:1577-89.
11. Blonde L, Merilainen M, Karwe V, Raskin P; TITRATE Study Group. Patient-directed titration for achieving glycaemic goals using a once-daily basal insulin analogue: an assessment of two different fasting plasma glucose targets. The TITRATE study. Diabetes Obes Metab 2009;11:623-31.

12. Diabetes Control and Complications Trial Research Group, Nathan DM, Genuth S, Lachin J, Cleary P, Crofford O, et al. The effect of intensive treatment of diabetes on the development and progression of long-term complications in insulindependent diabetes mellitus. N Engl J Med 1993;329:97786.

13. UK Prospective Diabetes Study (UKPDS) Group. Intensive blood-glucose control with sulphonylureas or insulin compared with conventional treatment and risk of complications in patients with type 2 diabetes (UKPDS 33). Lancet 1998; 352:837-53. 